Synthesis and Optical Properties of Photochromic Perinaphthothioindigo

N. J. Cherepy, R. D. Sanner

February 15, 2005 
This document was prepared as an account of work sponsored by an agency of the United States Government. Neither the United States Government nor the University of California nor any of their employees, makes any warranty, express or implied, or assumes any legal liability or responsibility for the accuracy, completeness, or usefulness of any information, apparatus, product, or process disclosed, or represents that its use would not infringe privately owned rights. Reference herein to any specific commercial product, process, or service by trade name, trademark, manufacturer, or otherwise, does not necessarily constitute or imply its endorsement, recommendation, or favoring by the United States Government or the University of California. The views and opinions of authors expressed herein do not necessarily state or reflect those of the United States Government or the University of California, and shall not be used for advertising or product endorsement purposes.

This work was performed under the auspices of the U.S. Department of Energy by University of California, Lawrence Livermore National Laboratory under Contract W-7405-Eng-48. 


\title{
Synthesis and Optical Properties of Photochromic Perinaphthothioindigo
}

\author{
Nerine J. Cherepy* and Robert D. Sanner
}

Chemistry and Materials Science Directorate, Lawrence Livermore National Laboratory,

Livermore, CA 94550

Article to be submitted to

Optical Materials

*Corresponding author: MS L-091, Lawrence Livermore National Laboratory, Livermore, CA 94550, Phone: (925) 424-3492, Fax: (925) 423-8772, cherepy1@1lnl.gov 


\begin{abstract}
(1,2-naphtho)(1,8-naphtho)thioindigo (PNT) has been synthesized following a simple FriedelCrafts route and its photochemical properties in toluene and PMMA characterized. PNT is a photochromic molecule capable of reversible photoisomerization between a yellow form (cisPNT, $\lambda_{\max }=484 \mathrm{~nm}$ ) and a purple form (trans-PNT, $\left.\lambda_{\max }=595 \mathrm{~nm}\right)$. The stable purple form converts to the yellow form with a trans-PNT to cis-PNT conversion quantum yield of 0.027 in toluene and 0.062 in PMMA. The unstable yellow form exhibits a cis-PNT to trans-PNT quantum efficiency of conversion of 0.27-0.85 in toluene and 0.17-0.68 PMMA, with highest conversion efficiency occurring in the vicinity of its $\lambda_{\max }$ of $484 \mathrm{~nm}$. Trans-PNT has a strong fluorescence quantum yield, 0.14 (toluene) and 0.16 (PMMA). For samples prepared photochemically in the cis-PNT form, slow thermal relaxation to the trans form occurs in the dark, with a half life of about 17 hours in toluene $\left(25^{\circ} \mathrm{C}\right)$ and even slower, 168 hours, in PMMA. The property of photoswitching between fluorescent and non-fluorescent forms makes this material a candidate for many applications in imaging and data storage. An anomalous excitation profile for the fluorescence from trans-PNT, showing a dip at $\sim 600 \mathrm{~nm}$, is an agreement with the wavelength-dependent quantum yield of conversion from trans-PNT to cis-PNT, which is highest at the absorbance peak.
\end{abstract}

Keywords: Photochromism, fluorescence data storage, fluorescence imaging, photochromic materials 


\section{INTRODUCTION}

Thioindigos are well-known photochromic dyes [Blanc, 1968;Wyman, 1973] with a reversible photochemical cis-trans isomerization. They also typically exhibit a higher quantum yield of conversion from the cis to the trans form, than from the trans to the cis form and a concomitant high fluorescence quantum yield from the trans form [Blanc, 1968]. The cis form typically has very low, nearly immeasurable intrinsic fluorescence. Perinaphthothioindigos, defined as containing at least one 1,8-naphtho group, are thioindigos whose trans absorption band is relatively red-shifted from its cis band, allowing good but not perfect photochemical separation of the cis and trans isomers. Furthermore, their fluorescence may be excited in the red, where inexpensive diode lasers are available. The photoswitching of bis(perinaphtho)thioindigo has been previously suggested for optical data storage and imaging [Takahashi, 1985; Irie, 1999].

Early efforts to implement thioindigoid optical elements were hindered by the overlap of the cis and trans absorption bands in most thioindigoid dyes. Thus, even with monochromatic light incident on the sample at the maximum absorption of either form, only photostationary states containing a mixture of the two isomers could be prepared. Perinaphthothioindigo dyes have been reported by several groups [Krysanov, 1981; Mostoslavskii, 1981; Klages, 1982a; Klages, 1982b; Veniaminov, 1986; Takahashi, 1985; Fukunishi, 1989; Irie, 1999]; the absorption spectra and properties differ among the dyes. Little information is available in the literature regarding synthesis of the perinaphthothioindigos: typically reference is made to a paper by Harley-Mason and Mann [Harley-Mason, 1942], which does not provide specific details on this synthesis. The form studied here, (1,2-naphtho)(1,8-naphtho)thioindigo, has an absorption spectrum which is in agreement with a compound called PNT by several groups [Krysanov, 
1981; Mostoslavskii, 1981; Veniaminov, 1986;]. Veniamanov and Lukov report that the trans form of PNT (Figure 1A) has a peak at $592 \mathrm{~nm}$ and the cis form (Figure 1B) has a peak at 480 $\mathrm{nm}$, while the bis(perinaphtho)thioindigo isomer shown in Figure 1C has $637 \mathrm{~nm}$ (trans) and 513 (cis) peaks. Fukunishi, et al. describe preparation of "PNTI"- bis[perinaphthothiopyran-(2)]indigo, $\mathrm{C}_{24} \mathrm{H}_{12} \mathrm{O}_{2} \mathrm{~S}_{2}, \mathrm{MW}=808$.4. They performed intramolecular Friedel-Crafts acylation of $\alpha$ naphthalenethioacetic acid, followed by oxidative dimerization. The structure of their compound is assumed to be that of Figure 1C based on the nomenclature used, although the reported molecular weight is more than twice as large as expected.

Photophysical measurements on perinaphthothioindigos have been reported in organic solvent solutions by Krysanov and Alfimov [Krysanov, 1981] and by Klages, Kobs and Memming [Klages, 1982a, Klages, 1982b]. These studies describe the excited state dynamics as follows. From the trans or cis ground singlet state, excitation promotes the molecule to an excited singlet state which intersystem crosses to a triplet intermediate. Klages and co-workers found decreased photoconversion quantum yields (trans to cis) at concentrations in excess of $2 \mathrm{x}$

$10^{-4} \mathrm{M}$. However, at $2 \times 10^{-4} \mathrm{M}$, they were able to generate a photostationary state of nearly pure cis isomer by irradiation at $617 \mathrm{~nm}$. They measured a $\Phi_{\text {conv }}$ (trans $\rightarrow$ cis) of $\sim 0.25$ at at $2 \times 10^{-4}$ M, with an extrapolated decrease to $\sim 0.01$ at $15 \times 10^{-4} \mathrm{M}$.

\section{MATerials ANd Methods}

\subsection{Synthesis of Perinaphthothioindigo.}

Preparation of 1-naphthylthioglycolic acid. $5.62 \mathrm{~g}$ (35.1 mmol) 1-naphthalene thiol (Acros Organics) and $70 \mathrm{~mL}$ of $95 \%$ ethanol were combined in a $250 \mathrm{~mL}$ round-bottom flask. The solution was warmed slightly and $3.5 \mathrm{~g}(87.5 \mathrm{mmol})$ sodium hydroxide and $10 \mathrm{~mL}$ of water 
were added to the mixture. $5.34 \mathrm{~g}(38.4 \mathrm{mmol})$ bromoacetic acid (Aldrich Chemical) was added dropwise with stirring and the mixture was heated at $60{ }^{\circ} \mathrm{C}$ for $2 \mathrm{~h}$. The reaction was then cooled to room temperature and stirred for $12 \mathrm{~h}$. The yellow reaction solution was then evaporated to dryness on a rotovap. $40 \mathrm{~mL}$ of water was added and the $\mathrm{pH}$ was adjusted to 2 with $5 \%$ aqueous hydrochloric acid. This solution was extracted two times with $40 \mathrm{~mL}$ portions of ethyl acetate and the combined ethyl acetate extracts were washed twice with $40 \mathrm{~mL}$ portions of saturated sodium chloride solution. The washed ethyl acetate extracts were dried over magnesium sulfate overnight. The dried extracts were filtered and evaporated to dryness on a rotovap. This crude product was recrystallized twice from hot diethyl ether, yielding $7.01 \mathrm{~g}$ of white crystals, $\mathrm{mp}$ $107-108^{\circ} \mathrm{C}(92 \%$ yield $)$.

Preparation of (1,2-Naphtho)(1,8-Naphtho)thioindigo (PNT). $1.00 \mathrm{~g}(4.59 \mathrm{mmol}) 1$ naphthylthioglycolic acid and $6 \mathrm{~mL}$ thionyl chloride (Aldrich Chemical) were heated to reflux for $0.5 \mathrm{~h}$ in a $15 \mathrm{~mL}$ round-bottom flask. The mixture was cooled and most of the thionyl chloride was removed on a rotovap. The resulting mixture was diluted to $6 \mathrm{~mL}$ with methylene chloride and added dropwise to a suspension of $1.41 \mathrm{~g}(10.6 \mathrm{mmol})$ aluminum chloride (Aldrich Chemical) in $18 \mathrm{~mL}$ methylene chloride in a $50 \mathrm{~mL}$ round-bottom flask maintained at $0{ }^{\circ} \mathrm{C}$ under a nitrogen atmosphere. This mixture was stirred for $0.5 \mathrm{~h}$ at $0{ }^{\circ} \mathrm{C}$ after all the acid chloride was added. The reaction solution was poured into $4.4 \mathrm{~g}$ potassium tartrate (Alfa Chemical) in $35 \mathrm{~mL}$ ice water and the resulting suspension was transferred to a separatory funnel. The methylene chloride layer was removed and the water layer was washed with three times with $15 \mathrm{~mL}$ portions of methylene chloride. All of the methylene chloride extracts were combined (approx. $60 \mathrm{~mL}$ ) and dried over sodium sulfate overnight. Removal of the methylene chloride on a rotovap yielded a green-black solid which was washed twice with cold diethyl ether and air 
dried. This material was subjected to column chromatography on silica gel with 3:1 methylene chloride:hexane as eluant. An intensely colored purple band was clearly visible as it moved down the column. This band was collected to give, after solvent removal, $0.40 \mathrm{~g}$ of dark violet product, mp $234-236^{\circ} \mathrm{C}$ (44\% yield).

\subsection{Mass spectrum of PNT.}

The mass spectrum was run on a Finnigan LCQ Advantage spectrometer using atmospheric chemical ionization. All masses are reported for the $\mathrm{M}+\mathrm{H}$ ion. The MS of the compound showed a parent peak at 397. MS/MS run on this 397 parent peak showed peaks at 369 (-CO), 364 (-SH), 353 (-CO2), 336 (-CO \& SH), 199 (parent /2), 171 (parent/2 -CO).

\subsection{NMR of PNT.}

The NMR was run on a Bruker Spectrospin $500 \mathrm{MHz}$ spectrometer using $\mathrm{CDCl}_{3}$ as

solvent. Chemical shifts are given in ppm relative to internal tetramethylsilane standard. ${ }^{1} \mathrm{H}$

NMR: $7.5-8.2$ (mult) (naphthalene ring protons). ${ }^{13} \mathrm{C}\left\{{ }^{1} \mathrm{H}\right\}$ NMR: 189.0, $190.0(\mathrm{C}=\mathrm{O}) ; 135.2$, $135.3(\mathrm{C}=\mathrm{C}) ; 124.4,124.9,126.2,126.3,126.4,126.6,127.0,127.1,127.2,127.6,128.0,128.5$, $128.8,129.0,129.3,129.4,129.8,131.1,133.4,133.6$ (naphthalene ring carbons).

\subsection{Preparation of Polymer Films.}

Polymethylmethacrylate (PMMA) films containing PNT were prepared by mixing solutions of PMMA (Aldrich Chemical, ave. molecular weight $=120,000$ ) in methylene chloride or toluene with PNT, pouring the solutions into a glass mold and allowing evaporation of the 
solvent. Solid pieces of high uniformity, clarity and smooth surfaces could be prepared in this way.

\subsection{Photochemical Characterization of Perinaphthothioindigo.}

Absorption spectra were acquired with a Perkin Elmer Lambda 19 Spectrophotometer, and fluorescence measurements were carried out with a Perkin Elmer LS-50B. For preparation of samples in the trans-dominant photostationary state, sample were exposed to white light from a Xenon lamp (300 $\mathrm{mW} / \mathrm{cm}^{2}$ at the exposure zone), samples were screened from IR by placing a beaker of water between the lamp and the samples. The cis-dominant photostationary state was prepared using the same setup, along with a LP-591 glass long-pass filter before the sample.

Fluorescence quantum yields were referenced to Nile Blue in ethanol $\left(\Phi_{\mathrm{fl}}=0.27\right)$ [Sens, 1981] and in PMMA to Rhodamine 6G $\left(\Phi_{\mathrm{fl}}=0.7\right)$ [Kurian, 2002]. Photoconversion quantum yields were determined by the method described for Aberchrome 540 [Kuhn, 2004], but using nitro-napthobenzoic acid $\left(\Phi_{\text {conv }}=0.5\right)$ [dvornikov, 1996] and malachite green $\left(\Phi_{\text {conv }}=1.0\right)$ as actinometers. The actinic light source was the tungsten halogen lamp in the LS-50B fluorimeter, and all measurements of solutions included the use of a magnetic stirring assembly (Starna Spinette, Starna Cells, Atascadero, CA) to ensure uniform irradiation and mixing. Solutions and PMMA samples were typically at $\mathrm{OD}=0.5-1.5$ at the irradiation wavelength (concentration of approximately $10^{-5} \mathrm{M}$, calculated using $\varepsilon_{595}=25,000 \mathrm{M}^{-1} \mathrm{~cm}^{-1}$ ), and no corrections were made for incomplete absorption of the light or for screening of actinic light by photoproducts, however, all measurements were performed in the low conversion regime. We estimate that the accuracy of our measurements is approximately $\pm 30 \%$. 


\section{RESULTS AND DisCUSSION}

The synthesis of the perinaphthothioindigo compound was accomplished by acylation of the naphthalene ring under Friedel-Crafts conditions, using the acid chloride of 1naphthylthioglycolic acid. This presumably leads to acylation at both the 2 and 8 position on the naphthalene ring [Oksengendler, 1959] to form 1,2- and 1,8-naphtho groups. Oxidation and dimerization of these intermediates readily occurs in air to form the naphthothioindigo product. Although three compounds could conceivably be formed from these two fragments, two symmetric and one asymmetric, we only see the asymmetric (1,2-naphtho)(1,8naphtho)thioindigo isomer under our product isolation conditions. Our visible spectrum agrees with that reported by Veniamanov and Lukov for PNT (structures A \& B in Figure 1). Specifically, we do not see the spectral bands reported for the symmetric isomer bis $(1,8$ naphtho)thioindigo (NTI, structure C) nor does our melting point or visible spectrum agree with that reported for the other symmetric isomer, bis(1,2-naphtho)thioindigo $\left(\mathrm{mp}=415^{\circ} \mathrm{C}\right.$ [Dziewonski, 1930], $\lambda_{\max }=565 \mathrm{~nm}$ [Friedlander, 1912]).

Two photostationary states may be formed, a cis-dominant state with absorption peak at $484 \mathrm{~nm}$ and a trans-dominant state, with absorption peak at $595 \mathrm{~nm}$. The quantum yield of fluorescence for the trans state was measured to be 0.14. As shown in Figure 3A, the absorption at $595 \mathrm{~nm}$ can be reduced by a factor of $27 \mathrm{x}$ by irradiation with a light filtered by a long pass LP591 filter. The fluorescence band at $640 \mathrm{~nm}$ can be reduced by a factor of 12x. Irradiation with monochromatic light at 595 produced a similar photostationary state. Complete conversion between the cis and trans states was not achievable in our experiments. However, in our studies of PNT we found that a strongly fluorescing photoproduct impurity builds up over time (after several weeks) in toluene. This photoproduct absorbs at $\sim 595 \mathrm{~nm}$ and fluoresces in the same 
region as trans-PNT. It is possible that even in very clean solutions some small amount of this photoproduct is present, resulting in the mismatch between the $\Delta \mathrm{A}$ and the $\Delta \mathrm{I}$ of fluorescence.

In PMMA, the photostationary states are more strongly mixed than in solution. Also, the $\Delta \mathrm{A}$ between the two states at $595 \mathrm{~nm}$ and the $\Delta \mathrm{I}$ at $640 \mathrm{~nm}$ with a $\Delta \mathrm{A}$ of $4.5 \mathrm{x}$ and a $\Delta \mathrm{I}$ of $3.0 \mathrm{x}$. The material is also fatigue resistant, as shown in Figure 4. A PMMA sample of PNT was cycled between the cis and trans forms using white light alternating with white light with an LP591 filter. Slight differences in illumination geometry and duration were effected, resulting in variations of depth of conversion per cycle. A PMMA sample of PNT was stored in a lit room for 1 year, and found to still exhibit photoswitching, however the dynamic range was slightly reduced to $2.5 \mathrm{x}$, compared to $3 \mathrm{x}$ in the freshly prepared sample.

Thioindigos are typically described as having trans forms that fluoresce and cis forms that are non-luminescent. However, we find that the with $480 \mathrm{~nm}$ excitation, the cis form has a very weak intrinsic fluorescence band at $550 \mathrm{~nm}$, with a quantum yield of 0.0035 . Figure 5 shows the luminescence excited at 480, as well as the luminescence excited at $595 \mathrm{~nm}$ for the cis-dominant PNT photostationary state. Such weak fluorescence from the cis-band is in good agreement with the high quantum yield of conversion from the cis to the trans form with excitation $<500 \mathrm{~nm}$.

The excitation profile of the trans-form luminescence surprisingly does not match its absorption spectrum (Figure 6). Our experiments show that excitation at $>620 \mathrm{~nm}$ has a very low quantum yield of photoconversion to cis- PNT, however, strong luminescence is excited, its emission matching that excited at $590 \mathrm{~nm}$. The transition thus excited at $>620 \mathrm{~nm}$ must have a very high quantum yield, and a very low absorbance strength. 
Quantum yields of conversion between the cis and trans forms measured in toluene and in PMMA are shown in Table 1. We measured the cis-to-trans conversion with $320 \mathrm{~nm}$ excitation, finding it to be highly efficient in both matrices, 0.27 in toluene and 0.17 in PMMA. The conversion quantum yield with $480 \mathrm{~nm}$ excitation, was measured to be even higher, 0.85 in toluene and 0.68 in PMMA. The trans-to-cis conversion measured with $590 \mathrm{~nm}$ excitation is much less efficient, 0.027 in toluene and 0.062 in PMMA. For our measurements in solution, concentrations were in the $1-8 \times 10^{-5} \mathrm{M}$ range, but many of the cited references omit this detail, shown by Klages et al. to be important,[klages refs] especially in the trans-to-cis conversion rate, thus the wide variation between measured values for the trans-to-cis conversion.

\section{SUMMARY}

We have measured photochemical properties of (1,2-naphtho)(1,8-naphtho)thioindigo (PNT) in toluene and PMMA. Trans- PNT is the stable isomer (purple in color), and exhibits strong fluorescence. In toluene, thermal conversion of cis- PNT to trans- PNT results in a halflife of about one day. However, in PMMA a yellow image (cis- PNT) created via exposure to red light was found to be stable and readable for more than 5 days, slowly fading to purple. CisPNT converts efficiently to trans- PNT under $480 \mathrm{~nm}$ excitation, while the conversion of transPNT to cis- PNT with excitation $>590 \mathrm{~nm}$ is about an order of magnitude less efficient. The properties of PNT may be used for information storage, in which cis- PNT is the unwritten form, $<500 \mathrm{~nm}$ light used to convert spatially distinct bits to trans- PNT, these bits subsequently read out through fluorescence excitation using light in the 590-650 nm range. 


\section{ACKNOWLEDGEMENTS}

We gratefully acknowledge the Laboratory Directed Research and Development program and the Non-Proliferation, Arms Control and International Security Directorate for support. Many thanks to Charles G. Stevens for initiating work on photochromic materials and for fruitful discussions. We acknowledge the assistance of Dr. Adam Love in obtaining the mass spectra and Dr. Robert Maxwell in obtaining the NMR spectra. This work was performed under the auspices of the U.S. Department of Energy by the University of California, Lawrence Livermore National Laboratory under contract No. W-7405-Eng-48. 


\section{REFERENCES}

(1) J. Blanc and D.L Ross., "A Procedure for Determining the Absorption Spectra of Mixed Photochromic Isomers Not Requiring their Separation,” J. Phys. Chem., 72, 2817-2824 (1968).

(2) G.M. Wyman, B.M., Zarnegar, "Excited State Chemistry of Indigoid Dyes. 1. Fluorescence vs. Cis-Trans Isomerization,” J. Phys. Chem., 77, 831-837 (1973).

(3) T. Takahashi, Y. Taniguchi, K. Umetani, H, Yokouchi, M. Hasimoto, Ts. Kano, "CisTrans Photoisomerization of Perinaphthothioindigo for Use as a Photo-Imaging Sensor Using Fluorescence under He-Ne Laser Excitation,” Jap. J. App. Phys., 24, 173-176 (1985).

(4) M. Irie, H. Ishida, T. Tsujioka, "Rewritable near-field optical recording on photochromic perinaphthothioindigo thin films: readout by fluorescence," Jpn., J. Appl. Phys., 38, 6114-6117, (1999).

(5) S. A. Krysanov and M. V. Alfimov, "Cis-trans photoisomerization of thioindigoid dyes studied by picosecond flash photolysis," Chem. Phys. Lett., 82, 51-54 (1981).

(6) M. A. Mostoslavskii, V. D. Paramonov, and V. F. Mandzhikov, "Direct photoisomerization of perithioindigoids," Ukrain. Khim. Zhur., 47, 440 (1981).

(7) C.P. Klages, K. Kobs, R. Memming, "Photocatalytec cis->trans Isomerization of Thioinidigoid Dyes,” Ber. Bunsenges. Phys. Chem., 86, 716-720 (1982).

(8) C.P. Klages, K. Kobs, R. Memming, "Cis->Trans Photoisomerization of Thioinidigoid Dyes." Chem. Phys. Lett., 90, 46-50. 
(9) A. V. Veniaminov and G. I. Lashkov, "Influence of polymer medium on photochemical isomerization of thioindigo compounds in polymethyl methacrylate," Vysokomolekulyarnye Soedineniya Seriya A, 28, 861-868 (1986).

(10) K. Fukunishi, M. Kobayashi, A. Morimoto, M. Kuwabara, H. Yamanaka, and M. Nomura, "Cis/trans isomerization of perinaphthothioindigo dye adsorbed on silica gel," Bull. Chem. Soc. Jpn., 62, 3733-3735 (1989).

(11) J. Harley-Mason and F. G. Mann, "The comparative reactivity of the carbonyl group in the thionaphthenquinones. Part I. The constitution of certain thioindigoid dyes," $J$. Chem. Soc., 404-415 (1942).

(12) R. Sens and K. H. Drexhage, "Fluorescence quantum yield of oxazine and carbazine laser dyes.," J. Luminesc., 24, 709-712, 1981.

(13) A. Kurian, N.A. George, B. Paul, V.P.N. Nampoori, C.P.G. Vallabhan, "Studies on Fluorescence Efficiency and Photodegradation of Rhodamine 6G Doped PMMA Using a Dual Beam Thermal Lens Technique," Laser Chem., 20, 99-110 (2002).

(14) H.J. Kuhn, S.E. Braslavsky, R. Schmidt, “Chemical Actinometry,” IUPAC report, 2004, www.iupac.org/publications/pac/2004/pdf/7612x2105.pdf.

(15) A.S. Dvornikov, I. Cokgor, F. McCormick, R. Piyaket, S. Esener., and P.M. Rentzepis, "Molecular transformation as a means for 3D optical memory devices," Opt. Comm., 128, 205-210 (1996).

(16) G. M. Oksengendler and E. P. Gendrikov, "Cyclization of S-(1-napthyl)thioglycolic acid," Ukrain. Khim. Zhur., 25, 206-209 (1959).

(17) Dziewonski, Bull. Acad. Pol. Sci. Ser. Chim., 198-201 (1930). 
(18) P. Friedlander and N. Woroshzow, "Concerning thioindigo dyes of the naphthalene series, ”Justus Liebig's Annalen der Chemie, 389, 1-23 (1912). 


\section{TABLeS}

Table 1. Photochemical rates and quantum yields for perinaphthothioindigos.

\begin{tabular}{|c|c|c|c|c|c|c|}
\hline & $\Phi_{\text {conv }}(\mathrm{c} \rightarrow \mathrm{t})$ & $\Phi_{\text {conv }}(\mathrm{t} \rightarrow \mathrm{c})$ & $\Phi_{\mathrm{fl}}(\mathrm{c})$ & $\Phi_{\mathrm{fl}}(\mathrm{t})$ & $\mathrm{k}_{\mathrm{th}}(\mathrm{c} \rightarrow \mathrm{t})(1 / \mathrm{s})$ & $\mathrm{t}_{1 / 2}(\mathrm{hr})$ \\
\hline $\begin{array}{c}\text { PNT, Toluene } \\
\text { (this work) }\end{array}$ & $0.27^{\mathrm{a}}$ & $0.027^{\mathrm{c}}$ & $0.0035^{\mathrm{b}}$ & & $1.1 \times 10^{-5}$ & \\
\hline $\begin{array}{c}\text { PNT, Butyl } \\
\text { acetate } \\
\text { (ref. 9) }\end{array}$ & $0.23^{\mathrm{d}}$ & $0.06^{\mathrm{d}}$ & & $0.03^{\mathrm{e}}$ & & \\
\hline $\begin{array}{c}\text { PNT, Toluene } \\
\text { (ref. 8) }\end{array}$ & $0.5^{\mathrm{e}}$ & $\begin{array}{c}0.25^{\mathrm{e}} \\
\text { at } 2 \times 10^{-4} \mathrm{M} \\
0.01 \text { at } 15 \times 10^{-4} \\
\mathrm{M}\end{array}$ & & & & \\
\hline $\begin{array}{c}\text { PNT in PMMA } \\
\text { (this work) }\end{array}$ & $0.17^{\mathrm{a}}$ & $0.062^{\mathrm{c}}$ & & $0.16^{\mathrm{c}}$ & $1.1 \times 10^{-6}$ & 168 \\
\hline $\begin{array}{c}\text { PNT in PMMA } \\
\text { (ref. 9) }\end{array}$ & & $0.00063^{\mathrm{d}}$ & & & \\
\hline $\begin{array}{c}\text { NTI, Benzene } \\
\text { (ref. 3) }\end{array}$ & $0.26^{\mathrm{f}}$ & $0.17^{\mathrm{d}}$ & & $0.07^{\mathrm{d}}$ & & \\
\hline $\begin{array}{c}\text { NTI, Benzene } \\
\text { (ref. 10) }\end{array}$ & $0.058^{\mathrm{g}}$ & $0.023^{\mathrm{h}}$ & & & $6.14 \times 10^{-6}$ & 31.3 \\
\hline
\end{tabular}

${ }^{\mathrm{a}} \lambda_{\text {conv }}=320 \mathrm{~nm}$

${ }^{\mathrm{b}} \lambda_{\text {conv }}=480 \mathrm{~nm}$

${ }^{c} \lambda_{\text {conv }}=590 \mathrm{~nm}$

d unspecified- interference filter, assumed to be at absorption peak

e unspecified dye laser, assumed to be at absorption peak

${ }^{\mathrm{f}} \lambda_{\text {conv }}=633 \mathrm{~nm}$

${ }^{\mathrm{g}} \lambda_{\text {conv }}=510 \mathrm{~nm}$

${ }^{\mathrm{h}} \lambda_{\text {conv }}=630 \mathrm{~nm}$ 


\section{Figure CAPTIONS}

Figure 1. (A) The perinaphthothioindigo studied here, PNT, trans form. (B) The perinaphthothioindigo studied here, PNT, cis form. (C) The perinaphthothioindigo studied in references $(\mathrm{X}, \mathrm{Y})$, referred to as NTI.

Figure 2. (A) Cuvette containing cis- PNT in toluene. (B) Cuvette containing trans- PNT. (C) Trans- PNT under white light illumination emits red light. (D) Cuvette containing cis- PNT, with a small zone of trans- PNT created at bottom of cuvette with a flash of white light, photograph taken 5 seconds after flash. (E) PNT in PMMA, showing image generated through a mask, using a LP591 filter and a fluorescent white light lamp, image fades slowly over several days.

Figure 3. (A) Absorption and fluorescence spectra of PNT in toluene. Two photostationary states may be formed, a cis-dominant state with absorption peak at $484 \mathrm{~nm}$ and a trans-dominant state, with absorption peak at $595 \mathrm{~nm}$. The fluorescence was excited $590 \mathrm{~nm}$. (B) Absorption and fluorescence spectra of PNT in PMMA. Separation between the cis- and trans-dominant photostationary states is less complete in PMMA.

Figure 4. Cycling between cis- and trans- PNT in PMMA shows proportional absorbance and fluorescence intensity over multiple cycles. 
Figure 5. The cis-state has a very weak intrinsic fluorescence band (emission maximum at 550 $\mathrm{nm})$, with a quantum yield of 0.0035 .

Figure 6. Excitation profiles for fluorescence from PNT (emission wavelength $710 \mathrm{~nm}$ ) shows that in addition to the trans state, a weakly absorbing state with an absorption maximum of $\sim 630$ $\mathrm{nm}$ produces strong luminescence. 


\section{FigURES}

(A)<smiles>O=C1/C(=c2\sc3c(=O)c4cccc(c24)C=CC=3)Sc2ccc3ccccc3c21</smiles>

Perinaphthothioindigo I (PNT)

Trans form (purple)
(B)

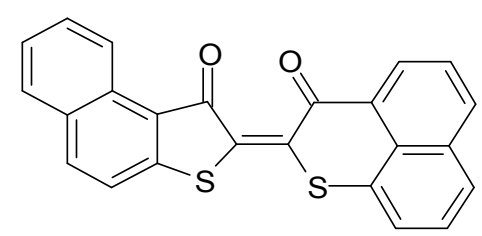

Perinaphthothioindigo I (PNT)

Cis form (yellow)
(C)

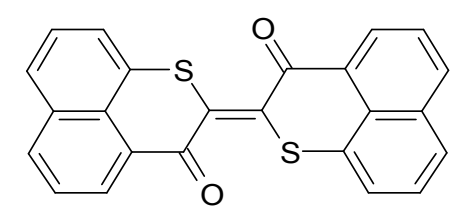

Perinaphthothioindigo II (NTI)

Figure 1. 

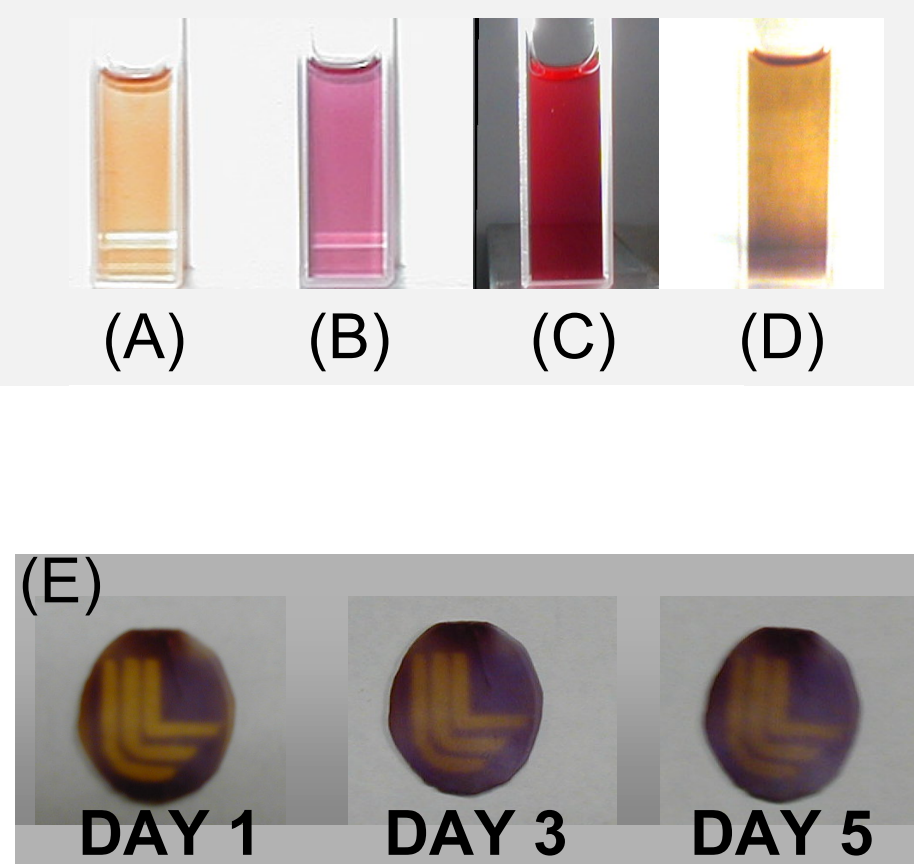

Figure 2. 


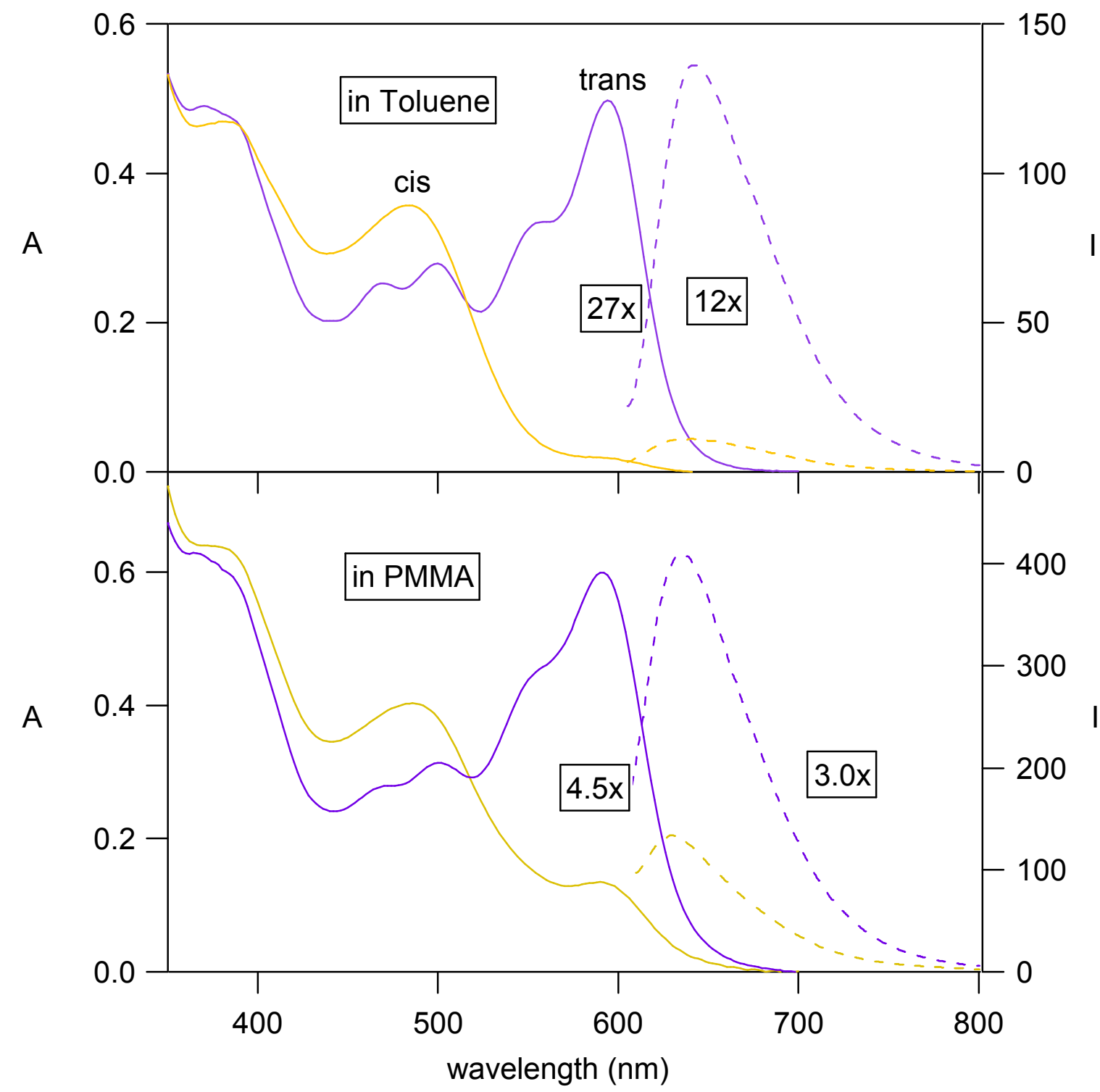

Figure 3. 


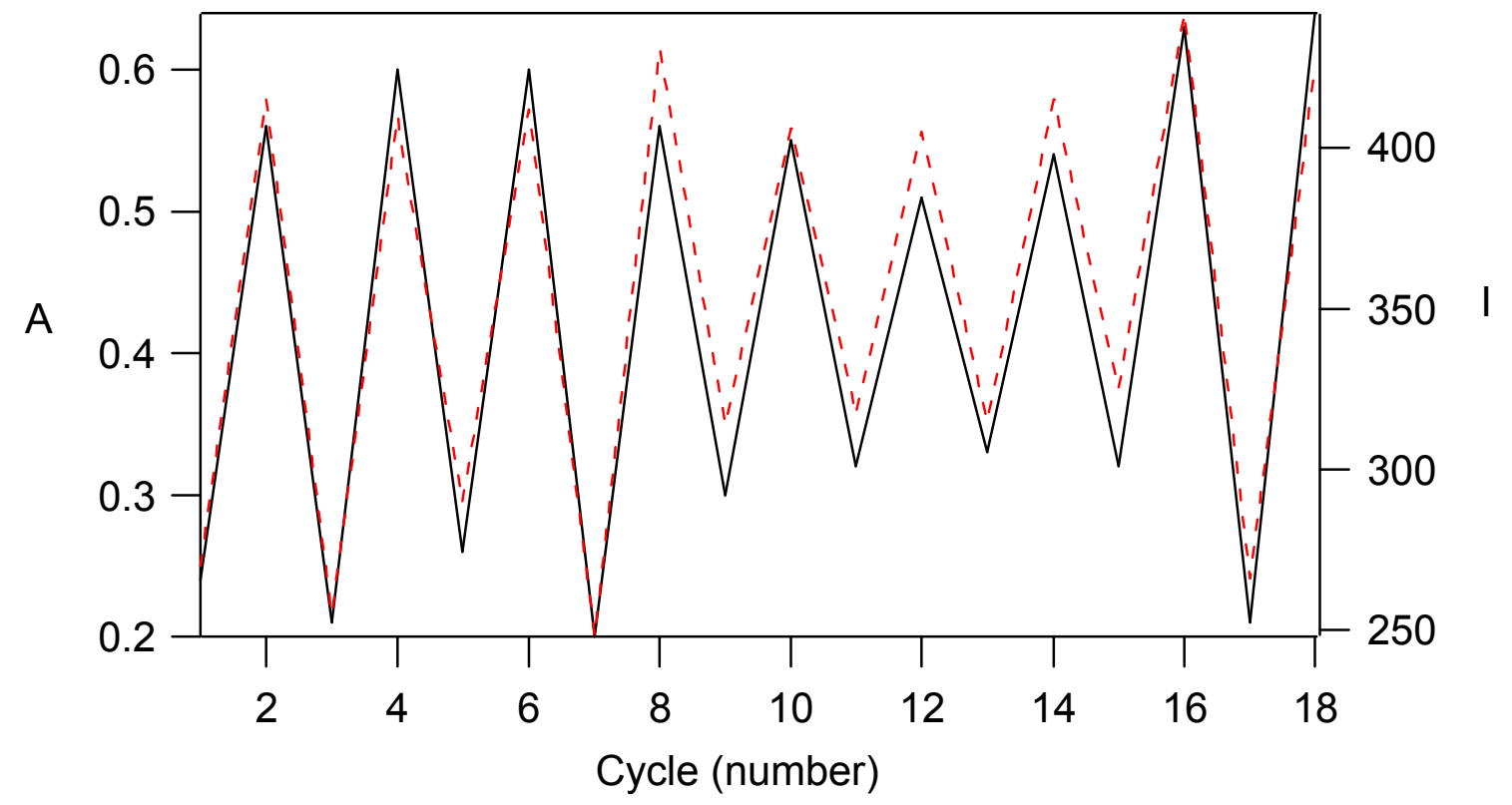

Figure 4. 


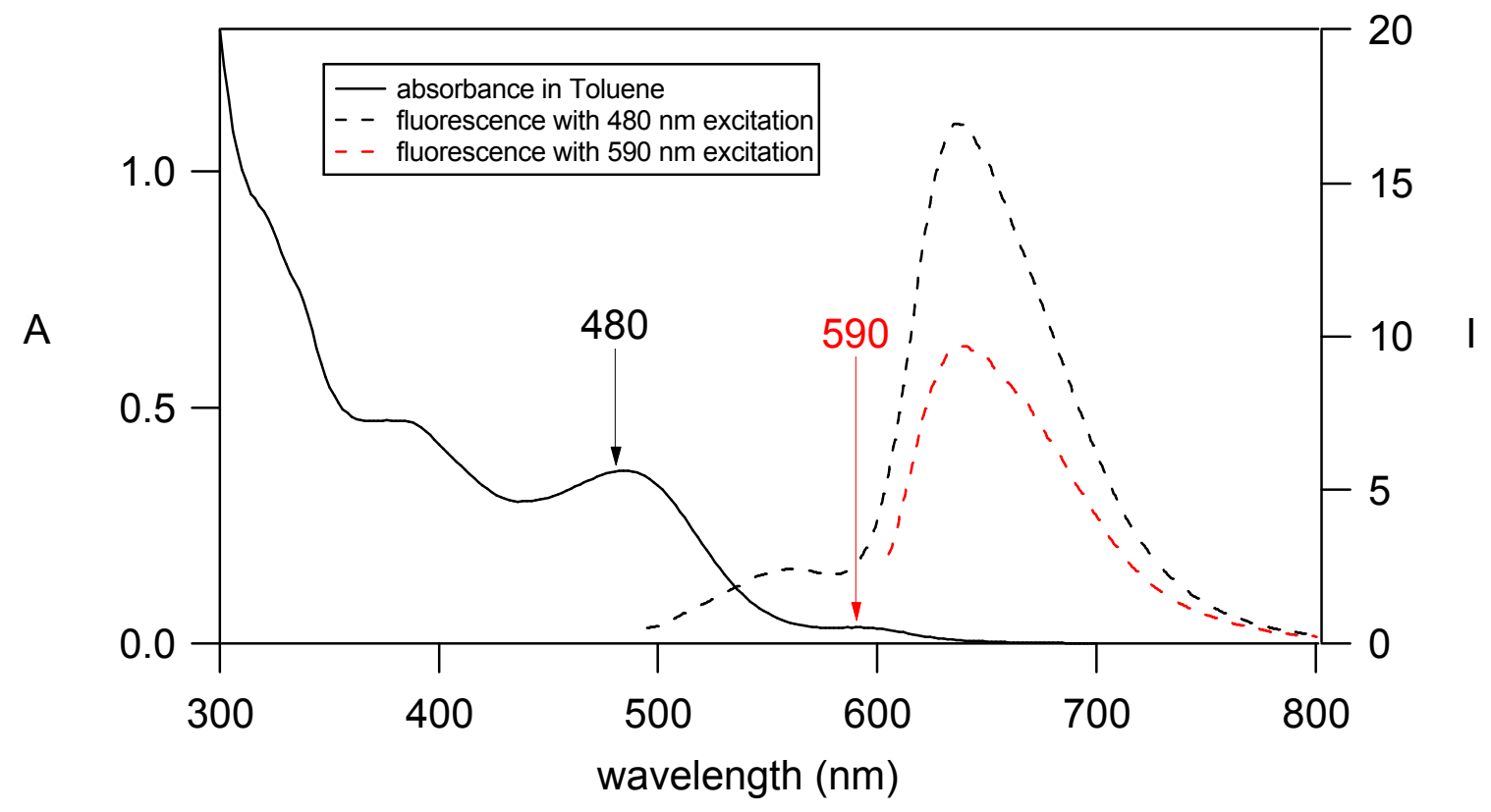

Figure 5. 


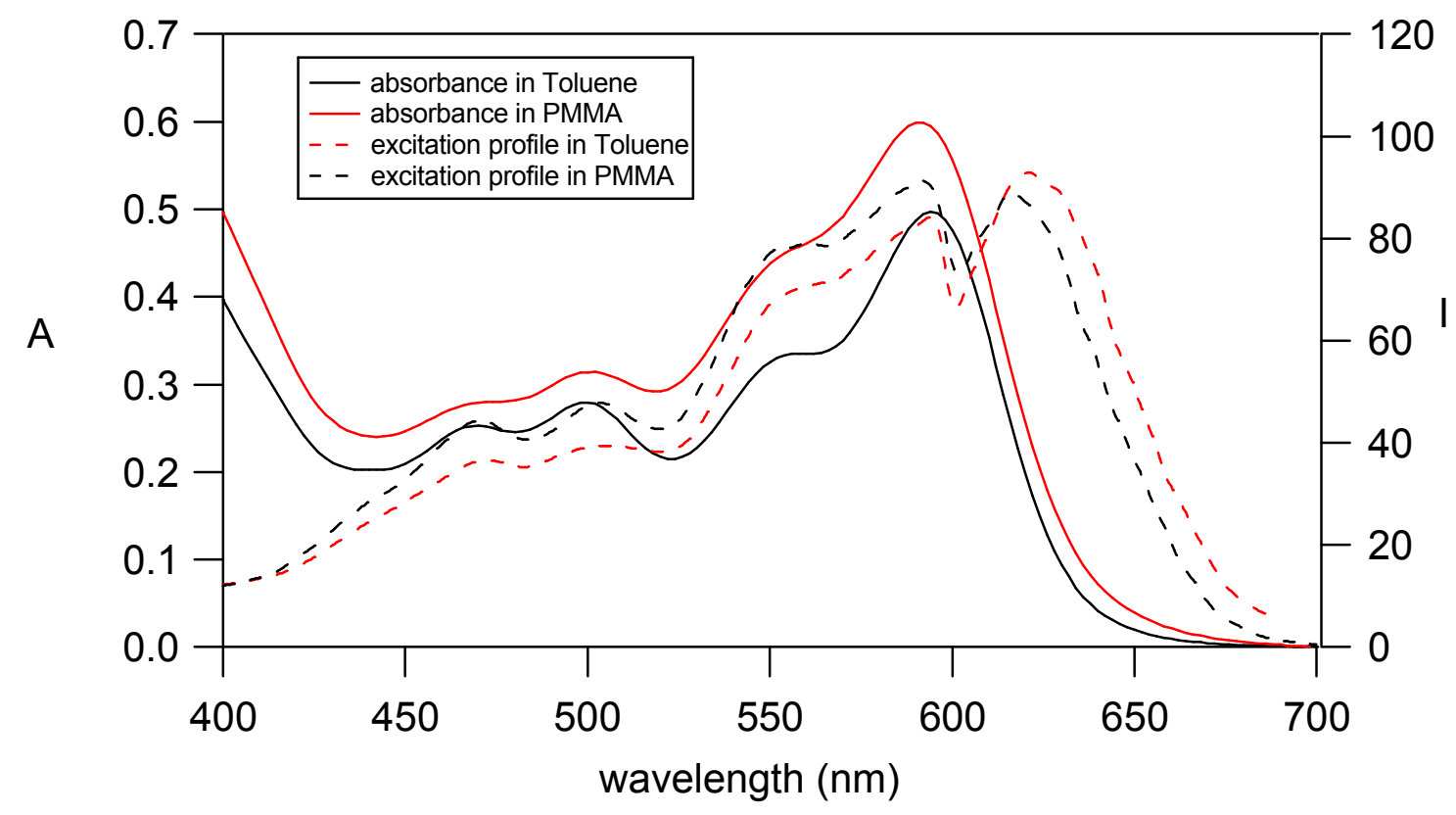

Figure 6. 